\title{
Soliton-Like Lamb Waves in Layered Media
}

\author{
I. Djeran-Maigre ${ }^{1}$ and S. V. Kuznetsov ${ }^{2}$ \\ ${ }^{1}$ University of Lyon, INSA Lyon, LGCIE, \\ 2Institute for Problems in Mechanics, Moscow, \\ ${ }^{1}$ France \\ ${ }^{2}$ Russia
}

\section{Introduction}

Solitons, or by the original terminology waves of translation, were for the first time observed and described by Scott Russel (1845) as a special kind of the hydrodynamic waves that can arise and propagate in narrow channels. Solitons are: (i) solitary waves, resembling propagation of the wave front of shock waves; (ii) these waves can propagate without considerable attenuation, or (iii) change of form; or (iv) diminution of their speed; see, Craik (2004). It was shown later on, that motion of these waves can be described by a non-linear $\mathrm{KdV}$ differential equation; see the work by the originators of the KdV-equation Korteweg and de Vries (1885) and the subsequent works by Lax (1968), Miles (1981), and Zwillinger (1997), where some of the analytical solutions are presented and the main properties of the KdV equation are analyzed.

Herein, we analyze the long-wave limits of Lamb waves propagating in multilayered elastic anisotropic plates at vanishing frequency $\omega \rightarrow 0$, or in terms of the wave number $r$, at $r \rightarrow 0$. These vanishing frequency Lamb waves satisfy conditions (i) - (iv), and thus, resemble the solitons. But, in contrast to the genuine solitons in hydrodynamics or their nonlinear analogues propagating in elastic solids; see, Eckl et al. (2004), Kawahara (1972), Kliakhandler, Porubov, and Verlande (2000), Planat and Hoummady (1989), Porubov et al. (1998), Samsonov (2001), our soliton-like waves are described by linear vectorial differential equations, known as the Christoffel equations for Lamb waves.

Studies of Lamb waves, as solutions of linear equations of motion for the infinite plates, and the corresponding soliton-like linear waves traveling with the finite phase speed at vanishing frequency have quite a long history. Presumably, the first asymptotic analysis of the waves propagating at vanishing frequency in an isotropic plate with the traction-free outer planes was performed by Gogoladze (1947). He obtained an analytical expression for the phase speed of such a wave by asymptotic analysis of the approximate equation of motion related to the theory of plates based on the Bernoulli - Euler hypotheses. Later on, the similar approach and a more elaborate one allowing to consider plates with different boundary conditions at outer planes, but still based on the approximated theories of plates, were exploited by Mindlin (1951a, b, 1958, 1960), Mindlin and Medick (1959), Mindlin and Onoe (1957), Onoe (1955), and Tolstoy and Usdin (1953). The latter authors reported highly intricate behavior of the disperse curves in the vicinity of the zero frequency. See also a more recent work by Pagneux and Maurel (2001), where the dispersion relations in the complex 
space were analyzed, and a paper by Kaplunov and Nolde (2002), where an asymptotic method was developed for analyzing the limiting case of the flexural mode. The behavior of the lowest branches of the disperse curves at $\omega \rightarrow 0$ for the traction-free isotropic plate was studied numerically by Lyon (1955), who used the classical theory of plates.

Along with the approximate theories of plates, a more general approach based on the Papkovich - Neuber elastic potentials for solving equations of motion was used. It allowed obtaining dispersion relations for different wave modes, not necessary flexural; see, Holden (1951). This approach was especially useful for analyzing behavior of the dispersion curves at $\omega \rightarrow 0$; see also works by Auld (1990) Ewing, Jardetzki, and Press (1957), Graff (1975). The Papkovich - Neuber potentials written in cylindrical coordinates allowed obtaining dispersion relations for elastic rods in the frame of Pochhammer - Chree theory for waves in isotropic rods; see, Pochhammer (1876), Chree (1889), Davies (1948), Meeker and Meitzler (1964), Miklowitz (1978), Mindlin and McNiven (1960), Onoe, McNiven, and Mindlin (1962). It should be noted that for rods a similar intricate behavior at $\omega \rightarrow 0$ of the lowest branches of the disperse curves for longitudinal and torsional waves was observed. The generalizations of the Papkovich - Neuber potentials to cover media with elastic anisotropy were also worked out; see Barber (2006), however the generalized potentials became so complicated that no analytical solutions obtained with them are known.

Analysis of Lamb waves propagating in anisotropic plates and their soliton-like counterparts relies on reducing the second-order vectorial equations of motion to the firstorder systems via different variants of the six-dimensional formalisms. Such a reduction can be referred to as the first step of the generalized Hamiltonian formalism; see Arnold (1989). From these formalisms the Stroh $(1958,1962)$ formalism is the most widely used, but there are also some other variants, among which we mention Lekhintskij (1963) formalism; see, also works by Barnett and Kirchner (1997) and Ting (1996, 1999, 2000) discussing equivalence of Stroh and Lekhnitskii formalisms. There are also different variants of the genuine six-dimensional Hamiltonian formalism applied to analysis of the surface acoustic wave; see, works by Tarn (2002a, b), Yan-ze Pen (2003), a recent paper by Fu (2007), and works by Kuznetsov (2002, 2003, and 2006). In the framework of the generalized Hamiltonian formalisms, several asymptotic approaches have been developed to study the limiting SH waves, propagating at $\omega \rightarrow 0$; see, Kuznetsov and Djeran-Maigre (2008); the lower modes of Lamb waves; see, Li and Romanowicz (1995); and the flexural modes of Lamb waves; see, Poncelet et al. (2006).

Another interesting variant of the asymptotic analysis is developed by Simonetti (2003), who studied behavior of propagation modes of Lamb and $\mathrm{SH}$ waves in a single-layered (infinite) plate with different types of boundary conditions by considering a two-layered plate and taking limits in material properties of one of the contacting layers.

Remarks 1.1. a) Analytical and numerical data; see Graff (1975), reveal that in the vicinity of the limiting phase speed $c_{s}$ the corresponding dispersion curve $c(\omega)$ satisfies a condition

$$
\left|c(\omega)-c_{s}\right|=O\left(\omega^{n}\right), \quad \omega \rightarrow+0,
$$

where $n>0$ is a positive number. However, by numerical analyses it is not possible to determine the exponent $n$. Below, a condition for obtaining the limiting speed $c_{s}$ will be developed. 
b) Low or vanishing frequencies of Lamb waves traveling with the phase speed satisfying condition (1.1), need in a small amount of energy needed for excitation. Indeed, the specific kinetic energy is determined by the following expression:

$$
E_{k i n} \equiv \frac{1}{2} \rho|\dot{\mathbf{u}}|^{2}=\frac{1}{2} \rho|\mathbf{m}|^{2} \omega^{2},
$$

where $\mathbf{m}$ is the wave amplitude (possibly varying along depth of a layer). The right-hand side of (1.2) ensures that at finite values of the amplitudes and at $\omega \rightarrow 0$, the specific kinetic energy vanishes. It can be shown that the specific potential energy is also proportional to square of amplitude and frequency, thus, vanishing at $\omega \rightarrow 0$, as well.

c) Importance of the limiting waves is underlined by the fact that they resemble propagation of the wave front (WF) in a layer; see Treves $(1982, \mathrm{Ch.V}, \$ 1)$ for definition of the WF and Achenbach (1973, Ch.IV, §4.5) for the corresponding notion used in acoustical applications. Following Lamb (1917), the displacement field of the wave traveling in an isotropic layer can be represented by the following

$$
\mathbf{u}(\mathbf{x}, t)=\left(\sum_{p=1}^{4} \mathbf{m}_{p} C_{p} e^{i r \gamma_{p} x^{\prime}}\right) e^{i r(\mathbf{n} \cdot \mathbf{x}-c t)},
$$

where $\mathbf{u}$ is the displacement field, and $\mathbf{m}_{p} \in \mathbb{R}^{3}$ are the unit amplitudes (polarizations). It is assumed that each vector $\mathbf{m}_{p}$ belongs to the sagittal plane. This plane is determined by the unit normal $\mathbf{w}=\mathbf{n} \times \mathbf{v}$, where $\mathbf{n}$ is the unit normal to the wave front and $\mathbf{v}$ is the unit normal to the median plane of the plate. In (1.3) $x^{\prime} \equiv \mathbf{v} \cdot \mathbf{x}$ is a coordinate along vector $v ; r$ is the wave number; $c$ is the phase speed; $t$ is time. The Christoffel parameters $\gamma_{p}$ will be introduced later on. In representation (1.3)

$$
\mathbf{u}^{p}(\mathbf{x}, t)=\mathbf{m}_{p} e^{i r \gamma_{p} x^{\prime}} e^{i r(\mathbf{n} \cdot \mathbf{x}-c t)}
$$

are the partial waves. The unknown coefficients $C_{p}$ in (1.3) are determined up to a multiplier by the traction-free boundary conditions:

$$
x^{\prime}= \pm h: \quad \mathbf{t}_{\mathbf{v}} \equiv \mathbf{v} \cdot \mathbf{C} \cdot \cdot \nabla_{\mathbf{x}} \mathbf{u}=0,
$$

where $\mathbf{C}$ is the fourth-order elasticity tensor (for isotropic medium tensor $\mathbf{C}$ is determined by two independent constants); and $2 h$ is the depth of a plate. Exponential multiplier $e^{i r(\mathbf{n} \cdot \mathbf{x}-c t)}$ in (1.3) and (1.4) stands for propagation of the plane wave front $\mathbf{n} \cdot \mathbf{x}=$ const .

Remark 1.2. Representation (1.3) is also valid in a case of anisotropic plate, provided: (A) the elasticity tensor has an axis of elastic symmetry, and (B) the wave travels in the direction of such an axis. Condition (A) is equivalent to monoclinic symmetry of the elasticity tensor, meaning that the elasticity tensor contains 13 independent decomposable components. At violating conditions (A) or (B), the amplitudes of partial waves may not belong to the sagittal plane. If that is the case, the six partial waves compose Lamb wave, instead of four partial waves used in (1.3); see, Kuznetsov (2002).

If a multilayered plate is concidered, the solution is usualy constructed by one of the following methods: (i) the transfer matrix (TM) method, known also as Thomson - Haskell method due to its originators; see, Thomson (1950), Haskell (1953) and more recent papers by Ryden et al. (2006) and Lowe (2008); and, (ii) the global matrix (GM) method; see, 
Knopoff (1964) and Mal and Knopoff (1968). The TM method is based on a sequential solution of the boundary-value problems on the interfaces and constructing the transfer matrices. The TM method will be discussed in a more detail in the subsequent sections. The GM method is based on solving a system of the governing differential equations with the piecewise constant coefficients, resulting in construction of the special "global matrix".

Herein, a variant of the modified TM (MTM) method will be developed. That is associated with construction of the fundamental exponential matrices and satisfying interface conditions in terms of these matrices. The MTM method allows us to analyze both phase speed and polarization of Lamb waves propagating at vanishing frequencies in anisotropic multilayered plates.

\section{Basic notations}

All the layers of a multilayered plate are assumed homogeneous and hyperelastic. Equations of motion for a homogeneous elastic anisotropic medium can be written in the following form

$$
\mathbf{A}\left(\partial_{x}, \partial_{t}\right) \mathbf{u} \equiv \operatorname{div}_{x} \mathbf{C} \cdot \cdot \nabla_{x} \mathbf{u}-\rho \ddot{\mathbf{u}}=0,
$$

where the elasticity tensor $\mathbf{C}$ is assumed to be positively definite:

$$
(\mathbf{A} \cdot \mathbf{C} \cdot \mathbf{A}) \equiv \sum_{i, j, m, n} A_{i j} C^{i j m n} A_{m n}>0, \quad \underset{\mathbf{A} \in \operatorname{sym}\left(R^{3} \otimes R^{3}\right), \mathbf{A} \neq 0}{\forall},
$$

In expression (2.2) $\operatorname{sym} \mathbf{A}=\frac{1}{2}\left(\mathbf{A}+\mathbf{A}^{t}\right)$.

Remark 2.1. For isotropic medium the positive definiteness of the elasticity tensor yields:

$$
\mu>0, \quad \lambda>-\frac{2}{3} \mu,
$$

where $\lambda$ and $\mu$ are Lamé constants.

Following Kuznetsov (2002, 2003) we consider a more general than (1.3) representation for Lamb waves, that is suitable for layers with arbitrary elastic anisotropy:

$$
\mathbf{u}(\mathbf{x}, t) \equiv \mathbf{f}\left(x^{\prime \prime}\right) e^{i r(\mathbf{n} \cdot \mathbf{x}-c t)},
$$

where $x^{\prime \prime}=i r x^{\prime}$ is a dimensionless coordinate; and $\mathbf{f}$ is the unknown vectorial function defining variation of the amplitude at the wave front. Substituting representation (2.3) into Eq. (2.1), yields the ordinary differential equation with respect to $\mathbf{f}$. This is known as the Christoffel equation for Lamb waves:

$$
-r^{2}\left(\mathbf{A}_{1} \partial_{x^{\prime \prime}}^{2}+\mathbf{A}_{2} \partial_{x^{\prime \prime}}+\mathbf{A}_{3}\right) \cdot \mathbf{f}=0
$$

where

$$
\mathbf{A}_{1}=\mathbf{v} \cdot \mathbf{C} \cdot \mathbf{v}, \quad \mathbf{A}_{2}=\mathbf{v} \cdot \mathbf{C} \cdot \mathbf{n}+\mathbf{n} \cdot \mathbf{C} \cdot \mathbf{v}, \quad \mathbf{A}_{3}=\mathbf{n} \cdot \mathbf{C} \cdot \mathbf{n}-\rho c^{2} \mathbf{I} .
$$

By introducing an auxiliary function $\mathbf{w}=\partial_{x^{\mathbf{f}}} \mathbf{f}$, Eq. (2.4) can be reduced to the matrix ODE of the first order: 


$$
\partial_{x^{\prime \prime}}\left(\begin{array}{l}
\mathbf{f} \\
\mathbf{w}
\end{array}\right)=\mathbf{G} \cdot\left(\begin{array}{l}
\mathbf{f} \\
\mathbf{w}
\end{array}\right),
$$

where $G$ is the matrix of the sixth rank for arbitrary elastic anisotropy, and of the fourth rank for the case described by conditions A and B in Remark 1.2:

$$
\mathbf{G}=\left(\begin{array}{cc}
\mathbf{0} & \mathbf{I} \\
-\mathbf{A}_{1}^{-1} \cdot \mathbf{A}_{3} & -\mathbf{A}_{1}^{-1} \cdot \mathbf{A}_{2}
\end{array}\right) .
$$

It can easily be deduced from (2.7)

$$
\operatorname{det}(\mathbf{G})=\operatorname{det}\left(\mathbf{A}_{3}\right) \cdot \operatorname{det}^{-1}\left(\mathbf{A}_{1}\right)
$$

In the right-hand side of (2.7) $\mathbf{0}$ and $\mathbf{I}$ are the corresponding $3 \times 3$ matrices. By means of (2.7), the general solution of Eq. (2.6) can be represented in the form

$$
\left(\begin{array}{l}
\mathbf{f} \\
\mathbf{w}
\end{array}\right)_{0}=e^{i r \mathbf{G} x^{\prime}} \cdot \vec{C},
$$

where $\vec{C}$ is the six-dimensional complex vector, defined up to a scalar multiplier by boundary conditions (1.5). Taking into account (2.9), representation (2.3) takes the form

$$
\left(\begin{array}{l}
\mathbf{u}(\mathbf{x}, t) \\
\mathbf{v}(\mathbf{x}, t)
\end{array}\right)=\left(e^{i r \mathbf{G} x^{\prime}} \cdot \vec{C}\right) e^{i r(\mathbf{n} \cdot \mathbf{x}-c t)},
$$

where $\mathbf{v}(\mathbf{x}, t)=\mathbf{w}\left(x^{\prime \prime}\right) e^{i r(\mathbf{n} \cdot \mathbf{x}-c t)}$.

Remarks 2.2. a) Representation (2.10) remains valid if matrix $\mathbf{G}$ is a non-semisimple matrix, i.e. when matrix $\mathbf{G}$ has Jordan blocks in its Jordan normal form.

b) Computing exponential matrix $e^{i r \mathbf{G} x^{\prime}}$ can be done by different numerical methods; see, Moler and Van Loan $(1978,2003)$ Higham (2001) and Zanna and Munthe-Kaas (2002), where different numerical schemes are discussed. For analytical purposes the exponential matrix can be constructed by applying two alternative methods: (1) the Taylor series expansion, or (2) reducing matrix $G$ to the Jordan canonical form and taking exponent of the diagonal matrix (assuming that $\mathbf{G}$ is a semisimple matrix)

$$
e^{i r \mathbf{G} x^{\prime}}=\mathbf{W}^{-1} \cdot e^{i r \mathbf{D} x^{\prime}} \cdot \mathbf{W} .
$$

where $\mathbf{D}$ is diagonal matrix, and $\mathbf{W}$ is a non-degenerate matrix needed to reduce $\mathbf{G}$ to the Jordan canonical form; see, Meyer (2002). If matrix G is not semisimple, representation (2.11) changes; see, Meyer (2002, \$7.3).

\section{Vanishing frequency Lamb wave in a homogeneous anisotropic plate}

Substituting solution (2.10) into boundary conditions (1.5) yields

$$
\mathbf{M} \cdot \vec{C}=0,
$$

where 


$$
\mathbf{M}=\left(\begin{array}{c}
\left(\mathbf{A}_{4}, \mathbf{A}_{1}\right) \cdot e^{+i r \mathbf{G} h} \\
-\left(\mathbf{A}_{4}, \mathbf{A}_{1}\right) \cdot e^{-i r \mathbf{G} h}
\end{array}\right)
$$

In (3.2)

$$
\mathbf{A}_{4}=\mathbf{v} \cdot \mathbf{C} \cdot \mathbf{n} .
$$

Existence of a non-trivial solution for Eq. (3.2) is equivalent to the following condition

$$
\operatorname{det}(\mathbf{M})=0
$$

Equation (3.4) is known as the dispersion equation for Lamb wave, since it implicitly defines speed of propagation as a function of frequency or wave number.

Proposition 3.1. At $r=0$ and at arbitrary anisotropy, Eq. (2.14) is trivially satisfied.

Proof flows out Eq. (3.2), which ensures at $r=0$ :

$$
\mathbf{M}=\left(\begin{array}{cc}
\mathbf{A}_{4} & \mathbf{A}_{1} \\
-\mathbf{A}_{4} & -\mathbf{A}_{1}
\end{array}\right) \text {. }
$$

It is clear that for matrix (3.5) condition (3.4) is satisfied.

However, the obtained at $r=0$ solution is meaningless; firstly, it does not satisfy Eq. (3.4) at small $r \rightarrow 0$; and secondly, it does not define speed of the wave at $r=0$. To construct the solution valid at $r=0$, the condition (1.1) will be used. Taking into account (3.4) and Proposition 3.1, condition (1.1) can be rewritten as a sequence of the following conditions imposed on the phase speed $c(r)$, that is implicitely defined by Eq. (3.4)

$$
\frac{d^{k}}{d r^{k}} c(r) \equiv-\left(\partial_{r}^{k} \operatorname{det}(\mathbf{M})\right) /\left.\left(\partial_{c} \operatorname{det}(\mathbf{M})\right)\right|_{r=0}=0, \quad k=1, \ldots, n .
$$

Conditions (3.6) are equivalent to

$$
\left.\partial_{r}^{k} \operatorname{det}(\mathbf{M})\right|_{r=0}=0, \quad k=1, \ldots, n .
$$

Taking Taylor's expansion (with respect to $r$ ) of the exponential mappings in (3.2), yields

$$
\begin{aligned}
& \mathbf{M}=\left(\begin{array}{rr}
\mathbf{A}_{4} & \mathbf{A}_{1} \\
-\mathbf{A}_{4} & -\mathbf{A}_{1}
\end{array}\right)+\frac{i r h}{1 !}\left(\begin{array}{ll}
-\mathbf{A}_{3} & \mathbf{A}_{4}-\mathbf{A}_{2} \\
-\mathbf{A}_{3} & \mathbf{A}_{4}-\mathbf{A}_{2}
\end{array}\right)+ \\
& +\frac{(i r h)^{2}}{2 !}\left(\begin{array}{rr}
-\mathbf{A}_{4} \mathbf{A}_{1}^{-1} \mathbf{A}_{3}+\mathbf{A}_{2} \mathbf{A}_{1}^{-1} \mathbf{A}_{3} & -\mathbf{A}_{4} \mathbf{A}_{1}^{-1} \mathbf{A}_{2}-\mathbf{A}_{3}+\mathbf{A}_{2} \mathbf{A}_{1}^{-1} \mathbf{A}_{2} \\
\mathbf{A}_{4} \mathbf{A}_{1}^{-1} \mathbf{A}_{3}-\mathbf{A}_{2} \mathbf{A}_{1}^{-1} \mathbf{A}_{3} & \mathbf{A}_{4} \mathbf{A}_{1}^{-1} \mathbf{A}_{2}+\mathbf{A}_{3}-\mathbf{A}_{2} \mathbf{A}_{1}^{-1} \mathbf{A}_{2}
\end{array}\right)+ \\
& +\frac{(i r h)^{3}}{3 !}\left(\begin{array}{ll}
\mathbf{A}_{4} \mathbf{A}_{1}^{-1} \mathbf{A}_{2} \mathbf{A}_{1}^{-1} \mathbf{A}_{3}+\mathbf{A}_{3} \mathbf{A}_{1}^{-1} \mathbf{A}_{3}- & -\mathbf{A}_{4} \mathbf{A}_{1}^{-1} \mathbf{A}_{3}+\mathbf{A}_{4}\left(\mathbf{A}_{1}^{-1} \mathbf{A}_{2}\right)^{2}+\mathbf{A}_{3} \mathbf{A}_{1}^{-1} \mathbf{A}_{2}+ \\
-\left(\mathbf{A}_{2} \mathbf{A}_{1}^{-1}\right)^{2} \mathbf{A}_{3} & +\mathbf{A}_{2} \mathbf{A}_{1}^{-1} \mathbf{A}_{3}-\left(\mathbf{A}_{2} \mathbf{A}_{1}^{-1}\right)^{2} \mathbf{A}_{2} \\
\mathbf{A}_{4} \mathbf{A}_{1}^{-1} \mathbf{A}_{2} \mathbf{A}_{1}^{-1} \mathbf{A}_{3}+\mathbf{A}_{3} \mathbf{A}_{1}^{-1} \mathbf{A}_{3}- & -\mathbf{A}_{4} \mathbf{A}_{1}^{-1} \mathbf{A}_{3}+\mathbf{A}_{4}\left(\mathbf{A}_{1}^{-1} \mathbf{A}_{2}\right)^{2}+\mathbf{A}_{3} \mathbf{A}_{1}^{-1} \mathbf{A}_{2}+ \\
-\left(\mathbf{A}_{2} \mathbf{A}_{1}^{-1}\right)^{2} \mathbf{A}_{3} & +\mathbf{A}_{2} \mathbf{A}_{1}^{-1} \mathbf{A}_{3}-\left(\mathbf{A}_{2} \mathbf{A}_{1}^{-1}\right)^{2} \mathbf{A}_{2}
\end{array}\right)+O\left(r^{4}\right)
\end{aligned}
$$


Substituting the first four terms of Taylor's series (3.8) into (3.7) and applying Schur's formulas; see, Meyer (2002), yields conditions (3.7) in the form

$$
\left.\left.\partial_{r}^{k} \operatorname{det}(\mathbf{M})\right|_{r=0} \equiv \partial_{r}^{k}\left(\operatorname{det}(\mathbf{W}) \operatorname{det}\left(\mathbf{Z}-\mathbf{X} \mathbf{W}^{-1} \mathbf{Y}\right)\right)\right|_{r=0}=0, \quad k=1, \ldots, n,
$$

where

$$
\begin{aligned}
\mathbf{W} & =\mathbf{A}_{4}-(i r h) \mathbf{A}_{3}+\frac{(i r h)^{2}}{2}\left(-\mathbf{A}_{4} \mathbf{A}_{1}^{-1} \mathbf{A}_{3}+\mathbf{A}_{2} \mathbf{A}_{1}^{-1} \mathbf{A}_{3}\right)+ \\
& +\frac{(i r h)^{3}}{3 !}\left(\mathbf{A}_{4} \mathbf{A}_{1}^{-1} \mathbf{A}_{2} \mathbf{A}_{1}^{-1} \mathbf{A}_{3}+\mathbf{A}_{3} \mathbf{A}_{1}^{-1} \mathbf{A}_{3}-\left(\mathbf{A}_{2} \mathbf{A}_{1}^{-1}\right)^{2} \mathbf{A}_{3}\right) \\
\mathbf{X} & =-\mathbf{A}_{4}-(i r h) \mathbf{A}_{3}+\frac{(i r h)^{2}}{2}\left(\mathbf{A}_{4} \mathbf{A}_{1}^{-1} \mathbf{A}_{3}-\mathbf{A}_{2} \mathbf{A}_{1}^{-1} \mathbf{A}_{3}\right)+ \\
& +\frac{(i r h)^{3}}{3 !}\left(\mathbf{A}_{4} \mathbf{A}_{1}^{-1} \mathbf{A}_{2} \mathbf{A}_{1}^{-1} \mathbf{A}_{3}+\mathbf{A}_{3} \mathbf{A}_{1}^{-1} \mathbf{A}_{3}-\left(\mathbf{A}_{2} \mathbf{A}_{1}^{-1}\right)^{2} \mathbf{A}_{3}\right) \\
\mathbf{Y} & =\mathbf{A}_{1}+(i r h)\left(\mathbf{A}_{4}-\mathbf{A}_{2}\right)+\frac{(i r h)^{2}}{2}\left(-\mathbf{A}_{4} \mathbf{A}_{1}^{-1} \mathbf{A}_{2}-\mathbf{A}_{3}+\mathbf{A}_{2} \mathbf{A}_{1}^{-1} \mathbf{A}_{2}\right)+ \\
& +\frac{(i r h)^{3}}{3 !}\left(-\mathbf{A}_{4} \mathbf{A}_{1}^{-1} \mathbf{A}_{3}+\mathbf{A}_{4}\left(\mathbf{A}_{1}^{-1} \mathbf{A}_{2}\right)^{2}+\mathbf{A}_{3} \mathbf{A}_{1}^{-1} \mathbf{A}_{2}+\mathbf{A}_{2} \mathbf{A}_{1}^{-1} \mathbf{A}_{3}-\left(\mathbf{A}_{2} \mathbf{A}_{1}^{-1}\right)^{2} \mathbf{A}_{2}\right) \\
\mathbf{Z} & =-\mathbf{A}_{1}+(i r h)\left(\mathbf{A}_{4}-\mathbf{A}_{2}\right)+\frac{(i r h)^{2}}{2}\left(\mathbf{A}_{4} \mathbf{A}_{1}^{-1} \mathbf{A}_{2}+\mathbf{A}_{3}-\mathbf{A}_{2} \mathbf{A}_{1}^{-1} \mathbf{A}_{2}\right)+ \\
& +\frac{(i r h)^{3}}{3 !}\left(-\mathbf{A}_{4} \mathbf{A}_{1}^{-1} \mathbf{A}_{3}+\mathbf{A}_{4}\left(\mathbf{A}_{1}^{-1} \mathbf{A}_{2}\right)^{2}+\mathbf{A}_{3} \mathbf{A}_{1}^{-1} \mathbf{A}_{2}+\mathbf{A}_{2} \mathbf{A}_{1}^{-1} \mathbf{A}_{3}-\left(\mathbf{A}_{2} \mathbf{A}_{1}^{-1}\right)^{2} \mathbf{A}_{2}\right)
\end{aligned}
$$

Matrices in (3.9) and (3.10) are correctly defined, if the phase speed $c$ does not coincide with any of the bulk wave speeds propagating in the direction of the wave normal $\mathbf{n}$. Henceforth, this is assumed to hold. Equations (3.9) are the necessary and sufficient conditions for existing a vanishing frequency Lamb wave that satisfies (1.1).

Remark 3.1. Parameter $n \geq 1$ in conditions (3.6) and (3.7) is dependent on anisotropy, and it characterizes attenuation of the phase speed $c(r)$ at $r \rightarrow 0$. Necessity of conditions (3.6) can be explained by analyzing Taylor's expansion of $\operatorname{det}(\mathbf{M})$ at small $r$, yielding

$$
\operatorname{det}(\mathbf{M})=r^{n} V_{n}+o\left(r^{n}\right), \quad r \rightarrow 0,
$$

where $V_{n}$ is an independent on $r$ constant. Taking into account (3.11), it becomes clear that conditions (3.6) and (3.7) define the phase speed, at which vanishes the lowest non-trivial coefficient $V_{n}$ of expansion (3.11).

\section{Vanishing frequency Lamb wave in a homogeneous isotropic plate}

For an isotropic elastic plate

$$
\begin{aligned}
& \mathbf{A}_{1}=(\lambda+2 \mu) \mathbf{v} \otimes \mathbf{v}+\mu(\mathbf{n} \otimes \mathbf{n}+\mathbf{w} \otimes \mathbf{w}), \quad \mathbf{A}_{2}=(\lambda+\mu)(\mathbf{v} \otimes \mathbf{n}+\mathbf{n} \otimes \mathbf{v}) \\
& \mathbf{A}_{3}=\left(\lambda+2 \mu-\rho c^{2}\right) \mathbf{n} \otimes \mathbf{n}+\left(\mu-\rho c^{2}\right)(\mathbf{v} \otimes \mathbf{v}+\mathbf{w} \otimes \mathbf{w}) \\
& \mathbf{A}_{4}=\lambda \mathbf{v} \otimes \mathbf{n}++\mu \mathbf{n} \otimes \mathbf{v}
\end{aligned}
$$


where $\mathbf{w}=\mathbf{v} \times \mathbf{n}$.

Substituting matrices (4.1) into (2.7) gives matrix $\mathbf{G}$ in a form

$$
\mathbf{G}=\left(\begin{array}{cccccc}
0 & 0 & 0 & 1 & 0 & 0 \\
0 & 0 & 0 & 0 & 1 & 0 \\
0 & 0 & 0 & 0 & 0 & 1 \\
\frac{\rho c^{2}-\mu}{\lambda+2 \mu} & 0 & 0 & 0 & -\frac{\lambda+\mu}{\lambda+2 \mu} & 0 \\
0 & \frac{\rho c^{2}-(\lambda+2 \mu)}{\mu} & 0 & -\frac{\lambda+\mu}{\mu} & 0 & 0 \\
0 & 0 & \frac{\rho c^{2}-\mu}{\mu} & 0 & 0 & 0
\end{array}\right) .
$$

For the isotropic plate the fundamental matrix $e^{i r \mathbf{G} x^{\prime}}$ can also be constructed explicitly by reducing matrix $\mathbf{G}$ to the Jordan normal form

$$
\mathbf{G}=\mathbf{W} \cdot \mathbf{D} \cdot \mathbf{W}^{-1},
$$

where $\mathbf{W}$ is a matrix containing (right) eigenvectors of matrix $\mathbf{G}$ stored columnwise

$$
\mathbf{W}=\left(\begin{array}{cccccc}
1 & 1 & 1 & 1 & 0 & 0 \\
-a & a & b^{-1} & -b^{-1} & 0 & 0 \\
0 & 0 & 0 & 0 & a^{-1} & -a^{-1} \\
a & -a & b & -b & 0 & 0 \\
-a^{2} & -a^{2} & 1 & 1 & 0 & 0 \\
0 & 0 & 0 & 0 & 1 & 1
\end{array}\right)
$$

and $\mathbf{D}$ is a diagonal matrix

$$
\mathbf{D}=\operatorname{diag}(a,-a, b,-b, a,-a) .
$$

In (4.4), (4.5) parameters $a$ and $b$ take the following values

$$
a=\sqrt{c^{2} / c_{S}^{2}-1}, \quad b=\sqrt{c^{2} / c_{P}^{2}-1}
$$

where

$$
c_{P}=\sqrt{\frac{\lambda+2 \mu}{\rho}}, \quad c_{S}=\sqrt{\frac{\mu}{\rho}}
$$

are speeds of bulk primary $\left(c_{P}\right)$ and secondary $\left(c_{S}\right)$ waves.

It can be proved that at any admissible values of $\lambda$ and $\mu$ satisfying condition (2.2'), matrix (4.2) is a semisimple matrix. Taking into account Eqs. (4.3) - (4.6) the fundamental matrix takes the form given by (2.11). Now, combining Eqs. (3.2) and (4.1 - (4.7), it is possible to represent matrix $\mathbf{M}$ in a complicated, but closed form. 
Considering Eqs. (4.2), (4.5), and (4.7), the dispersion equation (3.7) gives the following values for the phase speed of the vanishing frequency waves propagating in a homogeneous isotropic plate:

$$
c_{s_{1}}=2 \sqrt{\frac{\mu(\lambda+\mu)}{\rho(\lambda+2 \mu)}}, \quad c_{s_{2}}=\sqrt{\frac{\mu}{\rho}} .
$$

Remarks 4.1. a) Ewing, Jardetsky, and Press (1957) determined speed $c_{s_{1}}$ by applying asymptotic analysis based on Papkovich - Neuber potentials (and thus, confined to the isotropic plate only).

b) It can be shown from analyzing Eqs. (4.1) - (4.7) that a wave propagating with speed $c_{S_{2}}$ is polarized in direction normal to the sagittal plane (SH wave). Soliton-like $\mathrm{SH}$-waves were studied in (Kuznetsov and Djeran-Maigre, 2008).

c) The phase speed $c_{s_{1}}$ does not depend upon depth of the layer. Analysis of (4.8) shows, that at any admissible values of Lamés constants $\lambda$ and $\mu$, the speed $c_{s_{1}}$ lies in the interval $c_{T}^{\text {bulk }}<c_{s_{1}} \leq c_{L}^{\text {bulk }}$, where $c_{T}^{\text {bulk }}, c_{L}^{\text {bulk }}$ are speeds of the transverse and longitudinal bulk waves respectively. The phase speed $c_{s_{1}}$ coincides with $c_{L}^{\text {bulk }}$ only at $\lambda=0$.

d) At $c_{s_{1}}$ parameters $a$ and $b$ in (4.6) take the following values:

$$
a=\sqrt{\frac{3 \lambda+2 \mu}{\lambda+2 \mu}}, \quad b=i \frac{|\lambda|}{\lambda+2 \mu} .
$$

The inequality (2.2') ensures parameter $a$ in (4.9) to be real.

The eigenvectors (4.4) enable to obtain polarization of the vanishing frequency Lamb wave. Substituting the wave number $r=0$ and the phase speed $c=c_{s}$ into matrix $\mathbf{M}$, yields (up to a scalar constant) two eigenvectors $\vec{C}$ corresponding to the (multiple) zero-eigenvalue of matrix $\mathbf{M}$ :

$$
\vec{C}_{1}=(1,0,0,-1,0,0) ; \quad \vec{C}_{2}=\left(0,1,-\frac{\lambda}{\lambda+2 \mu}, 0,0,0\right) .
$$

The first eigenvector $\vec{C}_{1}$ ensures existence at $r=0$ the Lamb wave, linearly polarized in the $\mathbf{n}$-direction. Such a wave resembles the longitudinal bulk wave with respect to polarization, but naturally differs in the phase speed. According to (4.9) the second eigenvector in (4.10) also leads to a linearly polarized wave with the following complex (not normalized) amplitude:

$$
\mathbf{m}=\left(\frac{2 \mu}{\lambda+2 \mu}\right) \mathbf{v}+\left(\sqrt{\frac{3 \lambda+2 \mu}{\lambda+2 \mu}}+i \operatorname{sign}(\lambda)\right) \mathbf{n} .
$$

The real part of (4.11) leads to the slanted wave with respect to vectors $v$ and $\mathbf{n}$, while the imaginary part corresponds to a wave defined by the first eigenvector $\vec{C}_{1}$.

Since both $\vec{C}_{1}$ and $\vec{C}_{2}$ correspond to the zero eigenvalue, we can make a liner combination of them. This allows us to construct a vanishing frequency wave arbitrary (indefinitely) polarized in the sagittal plane. Summarizing, we arrive at 
Proposition 4.1. For the arbitrary isotropic traction-free plate and at $\omega \rightarrow 0$ there exists a nontrivial wave propagating with the phase speed $c_{s_{1}}$ independent of the thickness of a plate and indefinetely polarized in the sagittal plane.

\section{Vanishing frequency Lamb wave in a multilayered anisotropic plate}

At first a two-layered plate will be considered, and aftrewards the generalization to a plate with arbitrary number of anisotropic layeres will be given.

Let the two-layered plate consists of two homogeneous anisotropic layers with the ideal mechanical conact at the interface:

$$
\left\{\begin{array}{l}
\mathbf{u}\left(-h_{1}\right)=\mathbf{u}\left(+h_{2}\right) \\
\mathbf{t}_{-v}\left(-h_{1}\right)=-\mathbf{t}_{v}\left(+h_{2}\right)
\end{array},\right.
$$

where $2 h_{k}, k=1,2$ are the depths of the corresponding layers.

The outer surfaces of the plate are assumed to be traction-free:

$$
\left\{\begin{array}{l}
\mathbf{t}_{v}\left(+h_{1}\right)=0 \\
\mathbf{t}_{-v}\left(-h_{2}\right)=0
\end{array} .\right.
$$

By analogy with (2.10), the six-dimensional field in each of the layers can be represented in terms of the fundamental matrices $e^{i r \mathbf{G}_{k} x^{\prime}}$ :

$$
\left(\begin{array}{l}
\mathbf{u}_{k}(\mathbf{x}, t) \\
\mathbf{v}_{k}(\mathbf{x}, t)
\end{array}\right)=\left(e^{i r \mathbf{G}_{k} x^{\prime}} \cdot \vec{C}_{k}\right) e^{i r(\mathbf{n} \cdot \mathbf{x}-c t)} .
$$

Substituting representation (5.3) into interface conditions (5.1) yields:

$$
\left(\begin{array}{cc}
\mathbf{I} & \mathbf{0} \\
\left(\mathbf{A}_{4}\right)_{1} & \left(\mathbf{A}_{1}\right)_{1}
\end{array}\right) \cdot\left(e^{-i r \mathbf{G}_{1} h_{1}} \cdot \vec{C}_{1}\right)=\left(\begin{array}{cc}
\mathbf{I} & \mathbf{0} \\
\left(\mathbf{A}_{4}\right)_{2} & \left(\mathbf{A}_{1}\right)_{2}
\end{array}\right) \cdot\left(e^{+i r \mathbf{G}_{2} h_{2}} \cdot \vec{C}_{2}\right)
$$

It is easy to see that under condition of positive definiteness (2.2) for tensors $\mathbf{C}_{k}, k=1,2$, all $6 \times 6$ matrices appearing in (5.4), are non-degenerate. That allows us to represented the sixdimensional vector $\vec{C}_{2}$ in terms of $\vec{C}_{1}$ :

$$
\overrightarrow{\mathrm{C}}_{2}=\left(e^{-i r \mathbf{G}_{2} h_{2}}\right) \cdot\left(\begin{array}{cc}
\mathbf{I} & \mathbf{0} \\
\left(\mathbf{A}_{4}\right)_{2} & \left(\mathbf{A}_{1}\right)_{2}
\end{array}\right)^{-1} \cdot\left(\begin{array}{cc}
\mathbf{I} & \mathbf{0} \\
\left(\mathbf{A}_{4}\right)_{1} & \left(\mathbf{A}_{1}\right)_{1}
\end{array}\right) \cdot\left(e^{-i r \mathbf{G}_{1} h_{1}}\right) \cdot \vec{C}_{1}
$$

Remark 5.1. Expression (5.5) constitutes the basis of the Modified Transfer Matrix method, while the matrices appearing in the right-hand side of (5.5) are known as the transfer matrices.

Taking into account (5.5), the boundary conditions (5.2) can be expressed in the following form: 


$$
\mathbf{M} \cdot \vec{C}_{1}=0
$$

where $6 \times 6$ matrix $\mathbf{M}$ is

$$
\mathbf{M}=\left(\begin{array}{c}
\left(\left(\mathbf{A}_{4}\right)_{1},\left(\mathbf{A}_{1}\right)_{1}\right) \cdot\left(e^{+i r \mathbf{G}_{1} h_{1}}\right) \\
-\left(\left(\mathbf{A}_{4}\right)_{2},\left(\mathbf{A}_{1}\right)_{2}\right) \cdot\left(e^{-2 i r \mathbf{G}_{2} h_{2}}\right) \cdot\left(\begin{array}{cc}
\mathbf{I} & \mathbf{0} \\
\left(\mathbf{A}_{4}\right)_{2} & \left(\mathbf{A}_{1}\right)_{2}
\end{array}\right)^{-1} \cdot\left(\begin{array}{cc}
\mathbf{I} & \mathbf{0} \\
\left(\mathbf{A}_{4}\right)_{1} & \left(\mathbf{A}_{1}\right)_{1}
\end{array}\right) \cdot\left(e^{-i r \mathbf{G}_{1} h_{1}}\right)
\end{array}\right) .
$$

In (5.7) $\left(\left(\mathbf{A}_{4}\right)_{k},\left(\mathbf{A}_{1}\right)_{k}\right), k=1,2$ are $3 \times 6$ matrices. Existing (at $\left.r>0\right)$ the nontrivial solutions for Eq. (5.6) is equivalent to satisfying condition (3.4). However, for the vanishing frequency wave propagating at $r=0$, condition (3.4) becomes meaningless, as it was for a single homogeneous layer, for such a wave the additional conditions (3.6) should be applied to matrix (5.7).

For a plate consisting of $n>2$ homogeneous monoclinic layers in a contact, the secular matrix $\mathbf{M}$ becomes:

$$
\mathbf{M}=\left(\begin{array}{c}
\left(\left(\mathbf{A}_{4}\right)_{1},\left(\mathbf{A}_{1}\right)_{1}\right) \cdot\left(e^{+i r \mathbf{G}_{1} h_{1}}\right) \\
-\left(\left(\mathbf{A}_{4}\right)_{n},\left(\mathbf{A}_{1}\right)_{n}\right) \cdot \prod_{k=2}^{n}\left[\left(e^{-2 i r \mathbf{G}_{k} h_{k}}\right) \cdot\left(\begin{array}{cc}
\mathbf{I} & \mathbf{0} \\
\left(\mathbf{A}_{4}\right)_{k} & \left(\mathbf{A}_{1}\right)_{k}
\end{array}\right)^{-1} \cdot\left(\begin{array}{cc}
\mathbf{I} & \mathbf{0} \\
\left(\mathbf{A}_{4}\right)_{k-1} & \left(\mathbf{A}_{1}\right)_{k-1}
\end{array}\right)\right] \cdot\left(e^{-i r \mathbf{G}_{1} h_{1}}\right)
\end{array}\right)
$$

\section{Vanishing frequency Lamb wave in a multilayered isotropic plate}

Adopting the general method developed in the previous section and applying Eqs. (4.1) (4.7) to construct the fundamental matrices, we arrive at the following two values for the limiting phase speed:

$$
c_{s_{1}}=2 \sqrt{\left(\sum_{k=1}^{n} \mu_{k} h_{k} \frac{\lambda_{k}+\mu_{k}}{\lambda_{k}+2 \mu_{k}}\right) /\left(\sum_{k=1}^{n} \rho_{k} h_{k}\right)}, \quad c_{s_{2}}=\sqrt{\left(\sum_{k=1}^{n} \mu_{k} h_{k}\right) /\left(\sum_{k=1}^{n} \rho_{k} h_{k}\right)} .
$$

Analysing polarization of the corresponding waves reveals that a wave propagating with speed $c_{s_{1}}$ is polarized in the sagittal plane, whereas wave propagating with speed $c_{S_{2}}$ is a $\mathrm{SH}$ wave.

Confining ourselves to the genuine Lamb wave propagating with speed $c_{s_{1}}$, we can formulate: Proposition 6.1. a) Let $c_{s_{1}}^{\max }$ and $c_{s_{1}}^{\min }$ be maximal and minimal limiting wave speeds in the distinct layers (according to Proposition 4.1 these speeds are independent of thickness of the layers), then

$$
c_{s_{1}}^{\min } \leq c_{s_{1}} \leq c_{s_{1}}^{\max } .
$$

b) Supposing that depth of the $n$-th layer tends to infinity (halfspace) we arrive at the following value for the limiting wave speed

$$
c_{s_{1}}=2 \sqrt{\frac{\mu_{n}\left(\lambda_{n}+\mu_{n}\right)}{\rho_{n}\left(\lambda_{n}+2 \mu_{n}\right)}} .
$$


Proof a) flows out from considering the right-hand-side of (6.1), it ensures that all the terms

$$
\underset{k=1, \ldots, n}{\forall k} \frac{\mu_{k}\left(\lambda_{k}+\mu_{k}\right)}{\rho_{k}\left(\lambda_{k}+2 \mu_{k}\right)}>0
$$

are positive at the assumption of positive definiteness of the elasticity tensor. Proof $b$ ) also follows from the right-hand-side of (6.1) by passing to a limit at $h_{n} \rightarrow \infty$.

Remarks 6.1. a) Expression (6.1) for the limiting speed $c_{s_{1}}$ was apparently obtained for the first time; expression for the limiting speed $c_{s_{2}}$ was obtained by Kuznetsov (2006) and Kuznetsov and Djeran-Maigre (2008) with a different asymptotic scheme.

b) It follows from the right-hand side of (6.3) that the corresponding limiting speed is independent of physical and geometrical properties of other layers. It can be said that the limiting wave is insensitive to the layers of finite thickness in a contact with a halfspace.

c) Assuming in Eq. (6.1 $)_{1}$ that the plate is single-layered with $n=1$ and taking $\mu_{1}=1, \rho_{1}=1$, and $h_{1}=1$ we arrive at the following one-parametric expression for the speed $c_{s_{1}}$ :

$$
c_{s_{1}}=\frac{1}{\sqrt{2}} \sqrt{\frac{1}{1-v}}
$$

where $v$ is Poisson's ratio. The plot on Fig.1 shows variation of the longitudinal bulk wave speed and the limiting speed $c_{s_{1}}$ versus Poisson's ratio. The plot reveals that in the whole admissible range of $v \in(-1 ; 1 / 2)$, the speed $c_{s_{1}}$ remains substantially lower than the longitudinal bulk wave speed. The speed $c_{s_{1}}$ approaches speed of the shear bulk wave only at $v \rightarrow 1 / 2$, where actually $c_{s_{1}}=c_{S}$.

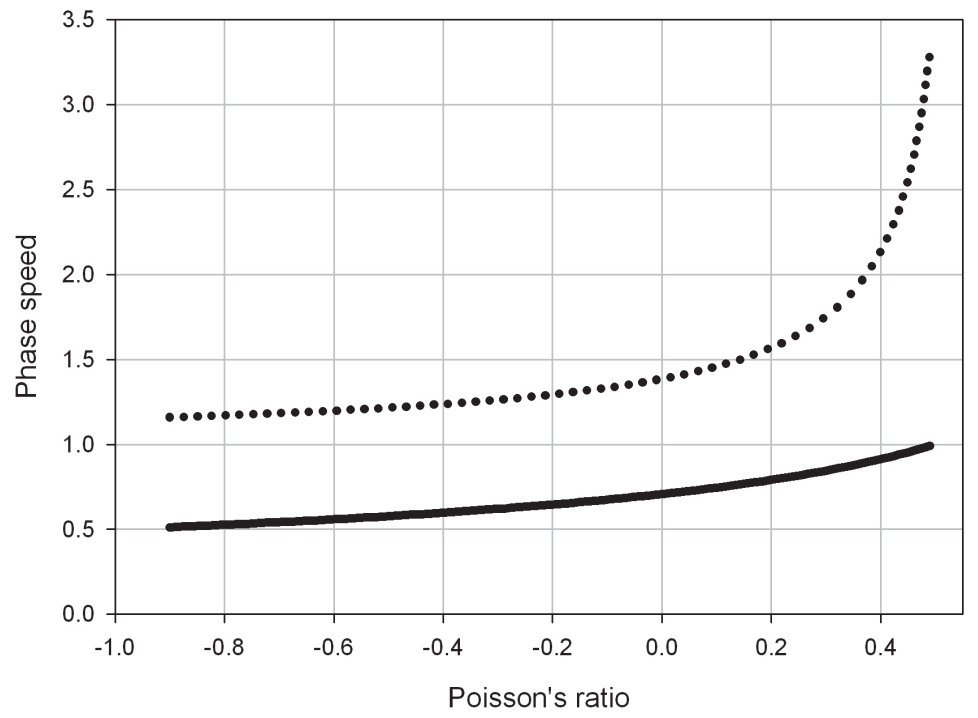

Fig. 1. Single layered isotropic plate: dependencies of the limiting speed $c_{s_{1}}$ (bold curve) and the longitudinal bulk wave speed (dotted curve) on Poisson's ratio. 
d) For a triple-layered plate with the outer layers of the same physical and geometrical properties (such a case often occurs in practice) the limiting speed $c_{s_{1}}$ is

$$
c_{s_{1}}=2 \sqrt{\left(2 \mu_{1} h_{1} \frac{\lambda_{1}+\mu_{1}}{\lambda_{1}+2 \mu_{1}}+\mu_{2} h_{2} \frac{\lambda_{2}+\mu_{2}}{\lambda_{2}+2 \mu_{2}}\right) /\left(2 \rho_{1} h_{1}+\rho_{2} h_{2}\right)}
$$

and $c_{s_{2}}$ is

$$
c_{s_{2}}=\sqrt{\frac{2 \mu_{1} h_{1}+\mu_{2} h_{2}}{2 \rho_{1} h_{1}+\rho_{2} h_{2}}},
$$

where index 1 is referred to the outer layers, and 2 corresponds to the inner layer. Assuming in Eq. (6.7) that $h_{1} \ll h_{2}$, while other physical properties of the layers have comparable values, yields coincidence of $c_{s_{2}}$ with the shear bulk wave speed of the inner layer.

Remarks 6.2. a) Expression (6.1) $)_{1}$ for the limiting speed $c_{s_{1}}$ was apparently obtained for the first time; expression for the limiting speed $c_{s_{2}}$ was obtained by Kuznetsov (2006) and Kuznetsov and Djeran-Maigre (2008) with a different asymptotic scheme.

\section{Acknowledgements}

Authors thank INSA de Lyon (France) and the Russian Foundation for Basic Research (Grants 08-08-00855 and 09-01-12063) for partial financial support.

\section{References}

Achenbach J.D. Wave Propagation in Elastic Solids, North-Holland Publ., Amsterdam London, 1973.

Arnol'd V.I. Mathematical Methods of Classical Mechanics, Springer-Verlag, N.Y., 1989.

Auld B.A. Acoustic Fields and waves in Solids, Vol.2, 2nd edition, Krieger Pub Co, Malabar FL, 1990.

Barber J.R. Three-dimensional elasticity solutions for isotropic and generally anisotropic bodies, Applied Mechanics and Materials, 5-6 (2006) 541-550.

Barber J.R. and Ting T.C.T. Three-dimensional solutions for general anisotropy, J. Mech. Phys. Solids, 55 (2007) 1993 - 2006

Chree $C$. The equations of an isotropic elastic solid in polar and cylindrical coordinates, their solutions and applications, Trans. Camb. phil. Soc. Math. Phys. Sci., 14 (1889) 250.

Craik A.D.D. The origins of water wave theory, Annual Review of Fluid Mechanics 36 (2004) $1-28$.

Davies R. M. A critical study of the Hopkinson pressure bar, Phil. Trans. R. Soc. A240 (1948) 375 - 457.

Eckl C., Kovalev A.S., Mayer A.P., Lomonosov A.M., and Hess P. Solitory surface acoustic waves, Physical Rev., E70 (2004) 1 - 15.

Ewing W.M., Jardetzky W.S., and Press F. Elastic Waves in Layered Media, McGraw-Hill Inc., N.Y., 1957.

Fu Y.B. Hamiltonian interpretation of the Stroh formalism in anisotropic elasticity, Proc. Roy. Soc. A. 463 (2007) 3073 - 3087. 
Gogoladze V.G. Dispersion of Rayleigh Waves in a Layer (in Russian), Publ. Inst. Seism. Acad. Sci. U.R.S.S. 119 (1947) 27 - 38.

Graff K. F. Wave Motion in Elastic Solids, Dover Inc., N.Y., 1975.

Hartman P. Ordinary differential equations, John Wiley \& Sons, Inc., N.Y., 1964.

Haskell N.A. Dispersion of surface waves on multilayered media, Bull. Seismol. Soc. America. 43 (1953) 17 - 34.

Higham N. J. Evaluating Padé approximants of the matrix logarithm, SIAM J. Matrix Anal. Appl., 22 (2001) 1126 - 1135.

Holden A.N. Longitudinal modes of elastic waves in isotropic cylinders and slabs, Bell Sys. Tech. J. 30 (1951) 956 - 969.

Kaplunov J.D. and Nolde, E.V. Long-Wave Vibrations of a Nearly Incompressible Isotropic Plate with Fixed Faces, Quart. J. Mech. Appl. Math. 55 (2002): 345 - 356.

Kawahara T. Oscillatory solitary waves in dispersive media, J. Phys. Soc. Japan 33 (1972) 260 $-268$.

Kliakhandler I.L., Porubov A.V., and Velarde M.G. Localized finite-amplitude disturbances and selection of solitary waves, Physical Review E 62 (2000) 4959-4962.

Korteweg D.J. and de Vries F. On the Change of Form of Long Waves Advancing in a Rectangular Canal, and on a New Type of Long Stationary Waves, Philosophical Magazine 39 (1895) $422-443$.

Knopoff L. A matrix method for elastic wave problems, Bull. Seismol. Soc. Am. 54 (1964) 431 -438 .

Kuznetsov S.V. Subsonic Lamb waves in anisotropic plates, Quart. Appl. Math. 60 (2002) 577 $-587$.

Kuznetsov S.V. Surface waves of non-Rayleigh type, Quart. Appl. Math. 61 (2003) 575 - 582.

Kuznetsov S.V. SH-waves in laminated plates, Quart. Appl. Math., 64 (2006) 153 - 165.

Kuznetsov S.V. and Djeran-Maigre I., Solitary SH waves in two layered traction free plates, Comptes Rendus Acad Sci., Paris, Ser. Mecanique, №336(2008) pp. 102 - 107.

Lamb H. On waves in an elastic plate, Proc. Roy. Soc. A93 (1917) $114-128$.

Lange J.N. Mode conversion in the long-wavelength limit, J. Acoust. Soc. Am., 41 (1967) 1449-1452.

Lax P. Integrals of Nonlinear Evolution Equations and Solitary Waves, Comm. Pure Appl. Math. 21 (1968) $467-490$.

Lekhnitskii S.G. Theory of Elasticity of an Anisotropic Elastic Body. Holden-Day, San Francisco 1963.

Li, X.-D., and Romanowicz B. Comparison of global waveform inversions with and without considering cross-branch modal coupling, Geophys. J. Int., 121 (1995) 695 - 709.

Lowe M.J.S. Matrix techniques for modeling ultrasonic waves in multilayered media, IEEE Transactions on Ultrasonics, Ferroelectrics, and Frequency Control 42 (1995) 525 542.

Lowe M.J.S. A computer model to study the properties of guided waves., J. Acoust. Soc. Am., $123(2008) 3654-3654$.

Lyon R.H. Response of an elastic plate to localized driving forces, J. Acoust. Soc. Am., 27 (1955) $259-265$.

Mal A.K., Knopoff L. A differential equation for surface waves in layers with varying thickness, J. Math. Anal. Appl. 21 (1968) 431 - 441. 
Meeker T.R. and Meitzler A.H. Guided wave propagation in elongated cylinders and plates, in: Physical Acoustics, (Ed. W. P. Mason) Vol. 1, Part A, Chap. 2. Academic Press, New York 1964.

Meyer C.D. Matrix Analysis and Applied Linear Algebra, SIAM, N.Y., 2002.

Miles J.W. The Korteweg-de Vries equation, a historical essay, J. Fluid Mech. 106 (1981) 131 147.

Miklowitz J. Elastic waves and waveguides, North-Holland, Amsterdam, 1978.

Mindlin R.D. The thickness shear and flexural vibrations of crystal plates. J. Appl. Phys. 22 (1951) 316.

Mindlin R.D. Influence of rotatory inertia and shear on flexural motions of isotropic, elastic plates, J. Appl. Mech., 18 (1951) 316.

Mindlin R.D. Vibrations of an infinite elastic plate and its cut-off frequencies, Proc Third US Nat. Congr. Appl. Mech., (1958) 225.

Mindlin R.D. Waves and vibrations in isotropic, elastic plates. In Structural Mechanics (Editors J.N. Goodier and N. Hoff). (1960) 199 - 232.

Mindlin R.D. and McNiven H.D. Axially symmetric waves in elastic rods, J. Appl. Mech., 27 (1960) $145-151$.

Mindlin R.D. and Medick M.A. Extensional vibrations of elastic plates, J. Appl. Mech., 26 (1959) $561-569$.

Mindlin R.D. and Onoe M. Mathematical theory of vibrations of elastic plates, In: Proceedings of the XI Annual Symposium on Frequency Control U. S. Army Signal Corps Engineering Laboratories, Fort Monmouth, New Jersey (1957) 17 - 40.

Moler C. and Van Loan Ch. Nineteen Dubious Ways to Compute the Exponential of a Matrix, SIAM Review, 20 (1978) 801 - 836.

Moler C. and Van Loan Ch. Nineteen Dubious Ways to Compute the Exponential of a Matrix, Twenty-Five Years Later SIAM Review, 45 (2003) 3 - 48.

Onoe M. A study of the branches of the velocity-dispersion equations of elastic plates and rods, Report: Joint Commitee on Ultrasonics of the Institute of Electrical Communication Engineers and the Acoustical Society of Japan, (1955) 1 - 21.

Onoe M., McNiven H.D., and Mindlin R.D. Dispersion of axially symmetric waves in elastic rods, J. Appl. Mech., 29 (1962) 729 - 734.

Pagneux V., Maurel A. Determination of Lamb mode eigenvalues, J. Acoust. Soc. Am., 110(3) (2001) Sep.

Planat M., Hoummady M. Observation of soliton-like envelope modulation generated in an anisotropic quartz plate by metallic in interdigital transducers, Appl. Phys. Lett, 55 (1989) 103 - 114.

Pochhammer L. Uber die Fortpflanzungsgeschwindigkeiten kleiner Schwingungen in einem unbegrenzten istropen Kreiszylinder, J. reine angew. Math. 81 (1876) 324 - 336.

Poncelet O., Shuvalov A.L., and Kaplunov J.D. Approximation of the flexural velocity branch in plates, Int. J. Solid Struct., 43 (2006) 6329 - 6346.

Porubov I.V., Samsonov A.M., Velarde M.G., and Bukhanovsky A.V. Strain solitary waves in an elastic rod embedded in another elastic external medium with sliding, Phys.Rev. Ser. E, 58 (1998) 3854 - 3864.

Russell Scott J. Report on waves, In: Fourteenth meeting of the British Association for the Advancement of Science, York, 1844 (London 1845), 311 - 390. 
Ryden N., Lowe M.J.S., Cawley P., and Park C.B. Evaluation of multilayered pavement structures from measurements of surface waves, Review of Progress in Quantitative Nondestructive Evaluation, 820 (2006) 1616 - 1623.

Simonetti F. Sound Propagation in Lossless Waveguides Coated with Attenuative Materials, PhD thesis, Imperial Colledge, 2003.

Soerensen M.P., Christiansen P.L., and Lomdahl P.S. Solitary waves on nonlinear elastic rods. I. J. Acoust. Soc. Amer., 76 (1984) 871 - 879.

Stroh A.N. Dislocations and cracks in anisotropic elasticity. Phil. Mag. 3 (1958) 625-646.

Stroh A.N. Steady state problems in anisotropic elasticity, J. Math. Phys. 41 (1962) 77 - 103.

Tanuma K. Stroh Formalism and Rayleigh Waves (Reprinted from J. Elasticity, 89, 2007), Springer, N.Y., 2007.

Tarn J.-Q. A state space formalism for anisotropic elasticity. Part. I. Rectilinear anisotropy. Int. J. Solids Structures, 39 (2002) 5157 - 5172.

Tarn J.-Q. A state space formalism for anisotropic elasticity. Part. II. Cylindrical anisotropy. Int. J. Solids Structures, 39 (2002) 5143 - 5155.

Thomson W.T. Transmission of elastic waves through a stratified solid medium, J. Appl. Phys. 21 (1950) 89 - 93.

Ting T.C.T. Anisotropic elasticity: theory and applications. Oxford University Press, New York, 1996.

Ting T.C.T. A modified Lekhnitskii formalism á la Stroh for anisotropic elasticity and classifications of the 6X6 matrix N, Proc. Roy. Soc. London, A455 (1999) 69 - 89.

Ting T.C.T. Recent developments in anisotropic elasticity. Int. J. Solids Structures, 37 (2000) $401-409$.

Ting T.C.T. and Barnett D.M. Classifications of surface waves in anisotropic elastic materials. Wave Motion 26 (1997) 207 - 218.

Tolstoy I. and Usdin E. Dispersive properties of stratified elastic and liquid media: a ray theory. Geophysics 18 (1953) $844-870$.

Treves F. Introduction to Pseudodifferential and Fourier Integral Operators, Vol.1, Plenum Press, N.Y. and London, 1982.

Yan-ze Peng Exact periodic wave solutions to a new Hamiltonian amplitude equation, J. Phys. Soc. Japan 72 (2003) 1356 - 1359.

Zanna A. and Munthe-Kaas H.Z. Generalized polar decompositions for the approximation of the matrix exponential, SIAM J. Matrix Anal. Appl., 23 (2002) 840 - 862.

Zwillinger D. Handbook of Differential Equations, Academic Press, Third Edition, 1998. 


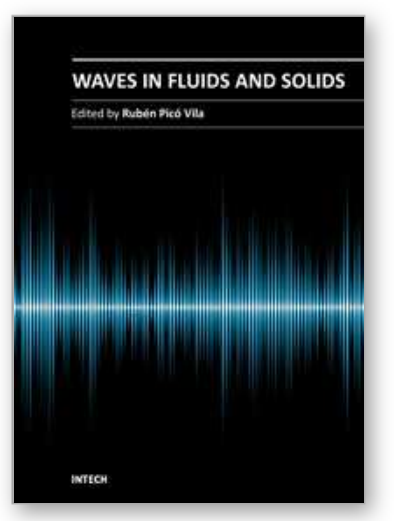

\author{
Waves in Fluids and Solids \\ Edited by Prof. Ruben Pico Vila
}

ISBN 978-953-307-285-2

Hard cover, 314 pages

Publisher InTech

Published online 22, September, 2011

Published in print edition September, 2011

Acoustics is an discipline that deals with many types of fields wave phenomena. Originally the field of Acoustics was consecrated to the sound, that is, the study of small pressure waves in air detected by the human ear. The scope of this field of physics has been extended to higher and lower frequencies and to higher intensity levels. Moreover, structural vibrations are also included in acoustics as a wave phenomena produced by elastic waves. This book is focused on acoustic waves in fluid media and elastic perturbations in heterogeneous media. Many different systems are analyzed in this book like layered media, solitons, piezoelectric substrates, crystalline systems, granular materials, interface waves, phononic crystals, acoustic levitation and soft media. Numerical methods are also presented as a fourth-order Runge-Kutta method and an inverse scattering method.

\title{
How to reference
}

In order to correctly reference this scholarly work, feel free to copy and paste the following:

I. Djeran-Maigre and S. V. Kuznetsov (2011). Soliton-Like Lamb Waves in Layered Media, Waves in Fluids and Solids, Prof. Ruben Pico Vila (Ed.), ISBN: 978-953-307-285-2, InTech, Available from: http://www.intechopen.com/books/waves-in-fluids-and-solids/soliton-like-lamb-waves-in-layered-media

\section{INTECH}

open science | open minds

\section{InTech Europe}

University Campus STeP Ri

Slavka Krautzeka 83/A

51000 Rijeka, Croatia

Phone: +385 (51) 770447

Fax: +385 (51) 686166

www.intechopen.com

\section{InTech China}

Unit 405, Office Block, Hotel Equatorial Shanghai

No.65, Yan An Road (West), Shanghai, 200040, China

中国上海市延安西路65号上海国际贵都大饭店办公楼 405 单元

Phone: +86-21-62489820

Fax: +86-21-62489821 
(C) 2011 The Author(s). Licensee IntechOpen. This chapter is distributed under the terms of the Creative Commons Attribution-NonCommercialShareAlike-3.0 License, which permits use, distribution and reproduction for non-commercial purposes, provided the original is properly cited and derivative works building on this content are distributed under the same license. 